\title{
COVID-19 Vaccine Hesitancy in the United States: A Systematic Review
}

Farah Yasmin ${ }^{1}$, Hala Najeeb ${ }^{1}$, Abdul Moeed ${ }^{1}$, Unaiza Naeem ${ }^{1 *}$, Muhammad Sohaib Asghar ${ }^{2}$, Najeeb Ullah Chughtai ${ }^{3}$, Zohaib Yousaf ${ }^{4}$, Binyam Tariku Seboka ${ }^{5}$, Irfan Ullah ${ }^{6}$, Chung-Ying Lin $^{7}$ and Amir H. Pakpour ${ }^{8}$

${ }^{1}$ Department of Internal Medicine, Dow University of Health Sciences, Karachi, Pakistan, ${ }^{2}$ Department of Internal Medicine, Dow University Ojha Hospital, Karachi, Pakistan, ${ }^{3}$ Department of General Surgery, Liaquat National Hospital and Medical College, Karachi, Pakistan, ${ }^{4}$ Department of Internal Medicine, Hamad Medical Corporation, Doha, Qatar, ${ }^{5}$ Department of Public Health, Dilla University, Dilla, Ethiopia, ${ }^{6}$ Department of Community Medicine, Kabir Medical College, Peshawar, Pakistan, ${ }^{7}$ Institute of Allied Health Sciences, National Cheng Kung University Hospital, College of Medicine, National Cheng Kung University, Tainan, Taiwan, ${ }^{8}$ Department of Nursing, School of Health and Welfare, Jönköping University, Jönköping, Sweden

\section{OPEN ACCESS}

Edited by:

Bijaya Kumar Padhi,

Post Graduate Institute of Medical

Education and Research (PGIMER), India

Reviewed by:

Kapil Goel,

Post Graduate Institute of Medical

Education and Research

(PGIMER), India

Krushna Chandra Sahoo,

Regional Medical Research Center (ICMR), India

*Correspondence: Unaiza Naeem

unaizanaeem.gs@gmail.com

Specialty section:

This article was submitted to Infectious Diseases - Surveillance, Prevention and Treatment, a section of the journal

Frontiers in Public Health

Received: 05 September 2021 Accepted: 01 November 2021 Published: 23 November 2021

Citation:

Yasmin F, Najeeb H, Moeed A, Naeem U, Asghar MS, Chughtai NU, Yousaf Z, Seboka BT, Ullah I, Lin C-Y and Pakpour AH (2021) COVID-19 Vaccine Hesitancy in the United States: A Systematic Review.

Front. Public Health 9:770985. doi: 10.3389/fpubh.2021.770985
Vaccine hesitancy in the US throughout the pandemic has revealed inconsistent results. This systematic review has compared COVID-19 vaccine uptake across US and investigated predictors of vaccine hesitancy and acceptance across different groups. A search of PUBMED database was conducted till 17th July, 2021. Articles that met the inclusion criteria were screened and 65 studies were selected for a quantitative analysis. The overall vaccine acceptance rate ranged from 12 to $91.4 \%$, the willingness of studies using the 10-point scale ranged from 3.58 to 5.12. Increased unwillingness toward COVID-19 vaccine and Black/African Americans were found to be correlated. Sex, race, age, education level, and income status were identified as determining factors of having a low or high COVID-19 vaccine uptake. A change in vaccine acceptance in the US population was observed in two studies, an increase of 10.8 and $7.4 \%$, respectively, between 2020 and 2021. Our results confirm that hesitancy exists in the US population, highest in Black/African Americans, pregnant or breastfeeding women, and low in the male sex. It is imperative for regulatory bodies to acknowledge these statistics and consequently, exert efforts to mitigate the burden of unvaccinated individuals and revise vaccine delivery plans, according to different vulnerable subgroups, across the country.

Keywords: COVID-19 vaccines, severe acute respiratory coronavirus 2 (SARS-CoV-2), vaccine hesitancy, vaccine acceptance, United States, intent to vaccinate

\section{INTRODUCTION}

Vaccines are critical in lowering disease-specific mortality rates (1) and the long-standing control of the COVID-19 pandemic is pivoted upon the development and uptake of the vaccine (2). Vaccines currently recommended and authorized in the US are BNT162b2 (Pfizer-BioNTech), mRNA1273 (Moderna), Johnson \& Johnson/Janssen (3) with the challenge now shifting from finding an effective cure to ensuring its implementation (4).

The US Centers for Disease Control and Prevention acclaims vaccination as one of the leading success stories of public health in the twentieth century (1) and a similar feat is now being aimed for the novel coronavirus. As part of Operation Warp Speed, the US administration alongside manufacturers and developers exerted efforts and by January 2021, hoped to deliver 300 million 
doses of a safe and effective vaccine for COVID-19 (5). By mid-January 2021, there were around 13 million persons who had COVID-19 vaccine initiated in the US (6) and while these numbers were promising, the vaccination process has been met with an undesirable, although not unusual, phenomenon wellknown as "vaccine hesitancy." Defined by the World Health Organization as "the delay in the acceptance or refusal to vaccinate despite the availability of vaccine services" (7), the term vaccine "hesitant" is preferred over "pro" and "anti" vaccination to avoid polarization and, as it insinuates that the minds can be persuaded toward acceptance (8).

Since the inception of vaccines in medical practice in the $1800 \mathrm{~s}$ (9), the public has not always concurred to getting vaccinated albeit many people have complied in face of apparent death and indisposition (1). Frequent outbreak of vaccine-preventable diseases in the US, such as 1,282 confirmed cases of measles in the United States in 2019 (8), can be ascribed to reduced vaccination and hence vaccine hesitancy, the latter ranked by the WHO as the top threat to global health in 2019 (1).

According to a global study, $72 \%$ of people would take the COVID-19 vaccine if deemed safe and effective, but willingness varies between countries (10). When studied under the framework of the $5 \mathrm{C}$ model of psychological antecedents that drive vaccine acceptance: confidence, constraints, complacency, calculation, and collective responsibility, the US population indicated $54 \%$ vaccine acceptance, a value that divulges vaccine skepticism (11). The US population has demonstrated inconsistent results over the period as between April 1-14 and November 25-December 8, 2020, the percentage who stated they were somewhat or very likely to get vaccinated declined from 74 to $56 \%$ (12). The percentage of US adults intending to get vaccinated has seen a $\mathrm{u}$-shaped pattern with results showing changes between September and December 2020, which correspond to pre-authorization and post-authorization dates in the US, respectively (7). Conversely, another national representative survey revealed the vaccine hesitancy to have a longitudinal decline of $10.8 \%$ points between October 2020 and March 2021 (13).

COVID-19 vaccine receptivity in the US has varied between states and subgroups. In mid-October 2020, acceptance rates ranged from $38 \%$ in the Northeast to $49 \%$ in the West (14). Vaccine hesitancy in the general population has been correlated with certain factors including gender, age, race, socioeconomic status, education level, and US-based surveys disclose important findings. Women have lower intentions than men to be vaccinated (14) and as of a study from April to December 2020, the self-reported likelihood of getting COVID-19 vaccination was lower among females than males (51 vs. 62\%) (12).

The cohort comprising of vaccine-hesitant individuals can be large enough to diminish the COVID-19 vaccine's potential to provide population immunity (15). Impediments to vaccination involve concerns including fear of side effects, inadequate information, short duration of immunity (14) forgoing vaccination due to lack of insurance or financial resources (16). Safety and effectiveness of the vaccine are the most pivotal detriments of hesitancy (4) while for some, especially marginalized factions, dissatisfaction with the health system owing to past experiences of discrimination, systematic racism deters them from vaccination (17).

Vaccine hesitancy is not a singular problem but attributed to various underlying causes that differ across time and communities (1). Recent results have shown somewhat reduced hesitancy, corresponding to the dates of vaccine approval and mass roll-out (13) and it is speculated that as the pandemic becomes more "real" to the Americans, vaccine acceptance can improve. Being one of the representative countries hardest hit by COVID-19, estimating vaccine hesitancy in the U.S could be important for future vaccine promotion and herd immunity (18). This systematic review aims to broaden the scope of discussion by studying factors coupled with vaccine hesitancy in the US population and the study's findings will be beneficial not only for COVID-19 vaccination coverage but also improving the existing healthcare system's preparedness for routine and emergency vaccination. Furthermore, our results will be imperative in helping strategize policies for tackling antagonism and for developing a thorough vaccine delivery plan.

\section{METHODS}

The review was performed following PRISMA guidelines. Papers published in MEDLINE (PubMed), Cochrane library, and Google Scholar assessing COVID-19 vaccine hesitancy/vaccine uptake/ vaccine acceptance in the English language were eligible for inclusion in the review. The inclusion criteria were: (1) peerreviewed published articles indexed in PubMed; (2) survey studies among the general population, healthcare workers, minority and religious communities, students, or patients (3) the major aim of the study was to evaluate COVID-19 vaccine acceptance/uptake/hesitancy in the US population only and (4) publication language was English. The exclusion criteria were: (1) the article did not aim to evaluate COVID-19 vaccine acceptance/hesitancy/uptake in the US population; (2) publication language was not English.

A search was carried out till 17th July 2021 using the following search strategy: (COVID * vaccine ${ }^{*}$ hesitancy [Title/Abstract]) OR (COVID * vaccine acceptance [Title/Abstract])) OR $\left(\right.$ COVID ${ }^{*}$ vaccine ${ }^{*}$ hesitancy [Title/Abstract])) OR (COVID * intention to vaccinate ${ }^{*}$ [Title/Abstract]) OR (COVID vaccine * accept ${ }^{*}[$ Title/Abstract] $)$.

Articles were screened by abstracts and titles. Studies shortlisted were cohort studies and cross-sectional studies that assessed COVID-19 vaccine acceptance over a certain time period, specific for each study, respectively. Studies drew comparison between vaccine acceptance and vaccine hesitance in the population surveyed. After selection, data extraction for the following items was conducted: title and date of the study, study period/duration, the target population of the study (e.g., general public, students, healthcare workers, patients, religious groups, and minority groups), region of US where the study took place, population characteristics i.e., sample size, \% female, mean age, $\%$ Whites, the definition of vaccine acceptance in the study, overall acceptance rate, acceptance rate by education level, factors relating to vaccine acceptance, and factors relating 


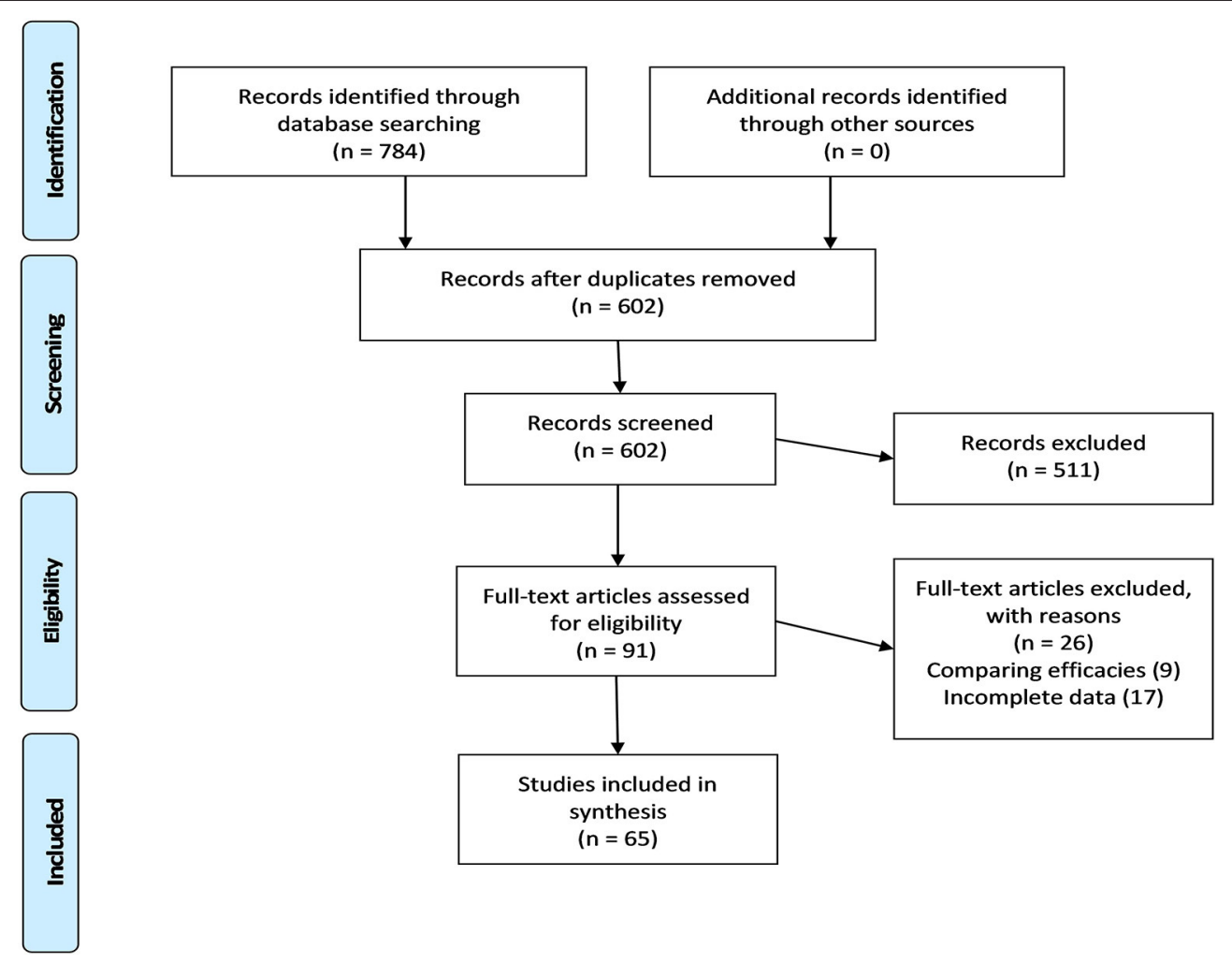

FIGURE 1 | PRISMA guidelines flow chart.

to vaccine hesitancy. Overall vaccine acceptance (\%) was taken from each study as deduced, corresponding to the definition of vaccine acceptance presented in the study. A forest plot was also constructed using Microsoft Excel version 2018 to demonstrate the overall prevalence of COVID-19 vaccine acceptance in the United States.

\section{RESULTS}

The search string provided in the Supplementary Material retrieved 784 records, of which 65 were included in the quantitative synthesis of observational studies on vaccine acceptance in the United States (US). Figure 1 illustrates the four-step selection process as per the PRISMA guidelines (19).

\section{Characteristics of the Literature}

The sample size of the selected studies ranged from 25 to 73,650 , with a total of $3,13,998$ individuals. Out of the 65 studies (67 surveys), 31 of them reported the study region, of which nine were conducted in New York City (NYC). Of the 50 studies reporting female sex \%, 38 of them had a percentage $>50$. Acceptance was measured as somewhat likely, extremely likely, and willingness to take the vaccine. The majority of the surveys that reported race and ethnicity, had a predominant nonHispanic white population. Vaccine acceptance was presented as percentages in 61 studies, whereas the remaining four studies reported means. Four of the studies used a point scale system to measure the likelihood of receiving the COVID-19 vaccine. Silva et al. (20) made use of a 10-point scale ranging from 1extremely unlikely and 10- extremely likely, whereas Meier et al. (21), Rhodes et al. (22), and Dorman et al. (7) used a 7-point scale. Detailed studies characteristics are present in Table 1.

\section{COVID-19 Vaccine Acceptance Rates}

Vaccine acceptance rates varied from a low of $12 \%$ to a high of $91.4 \%$. In 48 from 63 surveys, readiness to get vaccinated was $\geq 50 \%$. The mean and standard deviation of acceptance rate by Silva et al. was 7 (3.12); meanwhile, the willingness of studies using the 10-point scale ranged from 3.58 to 5.12 .

\section{COVID-19 Vaccine Acceptance Stratified per Region}

A total of 32 studies included the geographical location of the survey, comprising data of 20 states out of a possible 50. Overall, a low of $12 \%$ was observed in New York City in the Orthodox Jewish community, whereas a high of $90.1 \%$ was reported in Kansas among employees and students at the University of Kansas Medical Centre. Figure 2 illustrates the geographical average COVID-19 vaccine acceptance percentage reported in each state. Classifying vaccine acceptance per the four regions in the United States shows New England division of Northeast, West North Central of Midwest, Mountain division of West, and the whole of the South, except West South Central, had a low percentage of states with vaccine acceptance figures, however, 
TABLE 1 | Characteristics of the literature.

\begin{tabular}{|c|c|c|c|c|c|c|c|c|c|}
\hline Study & Study period & $\begin{array}{l}\text { Population } \\
\text { type/Settings }\end{array}$ & Region & $\begin{array}{l}\text { Sample size } \\
\text { (N) }\end{array}$ & Female sex \% & $\begin{array}{l}\text { White } \\
\text { population \% }\end{array}$ & $\begin{array}{l}\text { Acceptance } \\
\text { rate } \%\end{array}$ & $\begin{array}{l}\text { Predictors of } \\
\text { hesitance }\end{array}$ & $\begin{array}{l}\text { Predictors of } \\
\text { acceptance }\end{array}$ \\
\hline $\begin{array}{l}\text { Kobayashi et al. } \\
\text { (23) }\end{array}$ & $\begin{array}{l}\text { 13th-16th } \\
\text { November } 2020\end{array}$ & General & - & 661 & - & - & $90.00 \%$ & - & - \\
\hline Xiang et al. (24) & $\begin{array}{l}\text { 1st December-7th } \\
\text { January } 2021\end{array}$ & $\begin{array}{l}\text { Multiple sclerosis } \\
\text { patients }\end{array}$ & $\begin{array}{l}\text { Oregon and } \\
\text { Southwest } \\
\text { Washington }\end{array}$ & 410 & $23.90 \%$ & $89.50 \%$ & $70.10 \%$ & $\begin{array}{l}\text { Unsure about its } \\
\text { safety, developed } \\
\text { rapidly/possible } \\
\text { side effects }\end{array}$ & - \\
\hline Parente et al. (25) & $\begin{array}{l}\text { 14th August-28th } \\
\text { August } 2020\end{array}$ & $\begin{array}{l}\text { Employees or students } \\
\text { at a medical centre }\end{array}$ & Kansas & 3,292 & $78.00 \%$ & $87.60 \%$ & $90.10 \%$ & $\begin{array}{l}\text { Safety, side } \\
\text { effects, } \\
\text { Black/African } \\
\text { American }\end{array}$ & - \\
\hline Garcia et al. (26) & $\begin{array}{l}\text { 8th January-11th } \\
\text { February } 2021\end{array}$ & Dialysis center & - & 1,515 & $43.00 \%$ & $30.00 \%$ & $72.30 \%$ & $\begin{array}{l}\text { Black/African } \\
\text { American, } \\
\text { females }\end{array}$ & - \\
\hline $\begin{array}{l}\text { Thunstrom et al. } \\
\text { (27) }\end{array}$ & March 2020 & General & - & 3,133 & - & - & $80.50 \%$ & $\begin{array}{l}\text { Low trust in } \\
\text { government } \\
\text { agencies, females }\end{array}$ & - \\
\hline $\begin{array}{l}\text { Kecojevic et al. } \\
\text { (28) }\end{array}$ & $\begin{array}{l}\text { February-March } \\
2021\end{array}$ & Students & New Jersey & 352 & $73.60 \%$ & $49.50 \%$ & $52.80 \%$ & - & $\begin{array}{l}\text { Older age, } \\
\text { non-Hispanic } \\
\text { white, previous } \\
\text { illness, and } \\
\text { family member } \\
\text { vaccinated }\end{array}$ \\
\hline Yang et al. (29) & $\begin{array}{l}\text { December-January } \\
2021\end{array}$ & $\begin{array}{l}\text { Used tobacco or } \\
\text { marijuana }\end{array}$ & - & 387 & $53.50 \%$ & $74.90 \%$ & $49.10 \%$ & $\begin{array}{l}\text { Black/African } \\
\text { American }\end{array}$ & $\begin{array}{l}75.9 \% \text { of } \\
\text { willing receive } \\
\text { flu vaccine }\end{array}$ \\
\hline Carmody et al. (30) & $\begin{array}{l}\text { 7th December-20th } \\
\text { January } 2021\end{array}$ & Orthodox Jewish & NYC & 102 & $87.00 \%$ & - & $12.00 \%$ & $\begin{array}{l}\text { Natural infection } \\
\text { to be better than } \\
\text { vaccination for } \\
\text { developing } \\
\text { immunity }\end{array}$ & - \\
\hline Stoler et al. (31) & $\begin{array}{l}\text { 4th-17th June, } \\
2020\end{array}$ & General & - & 1,040 & - & - & $63.50 \%$ & $\begin{array}{l}\text { Black/African } \\
\text { American }\end{array}$ & Whites \\
\hline Serper et al. (32) & $\begin{array}{l}\text { 13th-23rd } \\
\text { December } 2020\end{array}$ & $\begin{array}{l}\text { Chronic disease } \\
\text { patients }\end{array}$ & $\begin{array}{l}\text { Philadelphia, } \\
\text { Pennsylvania }\end{array}$ & 1,215 & $50.00 \%$ & $86.50 \%$ & $85.00 \%$ & $\begin{array}{l}\text { Females, } \\
\text { Black/African } \\
\text { American, } \\
\text { lower-income, } \\
\text { and younger age }\end{array}$ & - \\
\hline Willis et al. (15) & July-August 2020 & General & Arkansas & 1,205 & $75.23 \%$ & $76.37 \%$ & $78.14 \%$ & $\begin{array}{l}\text { Black/African } \\
\text { American }\end{array}$ & - \\
\hline Lindholt et al. (11) & $\begin{array}{l}\text { 13th September } \\
\text { 2020-16th February } \\
2021\end{array}$ & General & - & 3,500 & - & - & $54.00 \%$ & - & - \\
\hline
\end{tabular}


TABLE 1 | Continued

\begin{tabular}{|c|c|c|c|c|c|c|c|c|c|}
\hline Study & Study period & $\begin{array}{l}\text { Population } \\
\text { type/Settings }\end{array}$ & Region & $\begin{array}{l}\text { Sample size } \\
\text { (N) }\end{array}$ & Female sex \% & $\begin{array}{l}\text { White } \\
\text { population } \%\end{array}$ & $\begin{array}{l}\text { Acceptance } \\
\text { rate } \%\end{array}$ & $\begin{array}{l}\text { Predictors of } \\
\text { hesitance }\end{array}$ & $\begin{array}{l}\text { Predictors of } \\
\text { acceptance }\end{array}$ \\
\hline $\begin{array}{l}\text { Nikolovski et al. } \\
\text { (33) }\end{array}$ & $\begin{array}{l}\text { 6th-20th November } \\
2020\end{array}$ & General & - & 7,402 & $46.20 \%$ & $91.80 \%$ & $91.30 \%$ & $\begin{array}{l}\text { Concerns about } \\
\text { its safety and } \\
\text { efficacy. Females, } \\
\text { Black/African } \\
\text { American }\end{array}$ & - \\
\hline $\begin{array}{l}\text { Thompson et al. } \\
\text { (34) }\end{array}$ & $\begin{array}{l}\text { June-December } \\
2020\end{array}$ & General & Michigan & 1,835 & $79 \%$ & $52 \%$ & $48 \%$ & $\begin{array}{l}\text { Black/African } \\
\text { American }\end{array}$ & - \\
\hline Ou et al. (35) & $\begin{array}{l}\text { 11th } \\
\text { November-2nd } \\
\text { December } 2020\end{array}$ & $\begin{array}{l}\text { Solid organ transplant } \\
\text { recipients and } \\
\text { non-SOTRs }\end{array}$ & - & 2,925 & $61.10 \%$ & $73.20 \%$ & $56.10 \%$ & $\begin{array}{l}\text { Lead to organ } \\
\text { transplant } \\
\text { rejection }\end{array}$ & \\
\hline Theis et al. (36) & $\begin{array}{l}\text { November } \\
\text { 2020-January } 2021\end{array}$ & $\begin{array}{l}\text { Individuals associated } \\
\text { with Wright-Patterson } \\
\text { Air Force Base }\end{array}$ & Dayton, Ohio & 816 & - & - & $77.33 \%$ & $\begin{array}{l}\text { Side effects, and } \\
\text { the spread of } \\
\text { misinformation }\end{array}$ & - \\
\hline Ciardi et al. (37) & $\begin{array}{l}\text { 10th December } \\
\text { 2020-5th January } \\
2021\end{array}$ & Healthcare workers & $\begin{array}{l}\text { The Bronx, New } \\
\text { York City, NY }\end{array}$ & 428 & $65 \%$ & $24 \%$ & $64 \%$ & - & - \\
\hline Liu et al. (18) & $\begin{array}{l}\text { 29th January-13th } \\
\text { February } 2021\end{array}$ & General & - & 3,702 & $51.80 \%$ & - & $72.20 \%$ & $\begin{array}{l}\text { Efficacy of } \\
\text { COVID-19 } \\
\text { vaccines, cost of } \\
\text { vaccination }\end{array}$ & - \\
\hline Gatwood et al. (38) & $\begin{array}{l}\text { 3rd-18th June } \\
2020\end{array}$ & General & Tennessee & 1,000 & $51 \%$ & $80.10 \%$ & $45.90 \%$ & $\begin{array}{l}\text { Effectiveness, } \\
\text { perceived lack of } \\
\text { disease risk, and } \\
\text { vaccine safety } \\
\text { concerns }\end{array}$ & - \\
\hline $\begin{array}{l}\text { Tsapepas et al. } \\
\text { (39) }\end{array}$ & March 2021 & $\begin{array}{l}\text { Kidney and pancreas } \\
\text { transplant recipients in } \\
\text { New York City }\end{array}$ & $\begin{array}{l}\text { New York City, } \\
\text { NY }\end{array}$ & 664 & $42.77 \%$ & $27.26 \%$ & $31.63 \%$ & $\begin{array}{l}\text { Younger age, } \\
\text { Black/African } \\
\text { American, } \\
\text { medium level of } \\
\text { poverty, safety in } \\
\text { transplant } \\
\text { recipients }\end{array}$ & - \\
\hline Bass et al. (17) & $\begin{array}{l}\text { 15th May-6th July } \\
2020\end{array}$ & General & $\begin{array}{l}\text { Miami, FL, New } \\
\text { York City, NY, } \\
\text { San Francisco, } \\
\text { CA }\end{array}$ & 501 & $36.00 \%$ & $24.00 \%$ & $87.00 \%$ & $\begin{array}{l}\text { Younger age, } \\
\text { females high } \\
\text { school education, } \\
\text { lower-income, } \\
\text { Black/African } \\
\text { American }\end{array}$ & - \\
\hline Johnson et al. (40) & $\begin{array}{l}\text { 15th } \\
\text { September-4th } \\
\text { December } 2020\end{array}$ & General & Louisiana & 248 & $57 \%$ & (Black 65.2\%) & $32.66 \%$ & $\begin{array}{l}\text { Side effects, } \\
\text { females, } \\
\text { Black/African } \\
\text { American, high } \\
\text { school, older age }\end{array}$ & - \\
\hline
\end{tabular}


TABLE 1 | Continued

\begin{tabular}{|c|c|c|c|c|c|c|c|c|c|}
\hline Study & Study period & $\begin{array}{l}\text { Population } \\
\text { type/Settings }\end{array}$ & Region & $\begin{array}{l}\text { Sample size } \\
\text { (N) }\end{array}$ & Female sex \% & $\begin{array}{l}\text { White } \\
\text { population \% }\end{array}$ & $\begin{array}{l}\text { Acceptance } \\
\text { rate } \%\end{array}$ & $\begin{array}{l}\text { Predictors of } \\
\text { hesitance }\end{array}$ & $\begin{array}{l}\text { Predictors of } \\
\text { acceptance }\end{array}$ \\
\hline Daly et al. (13) & October 2020 & General & - & 6,016 & - & - & $54.00 \%$ & $\begin{array}{l}\text { Younger age } \\
\text { Females. } \\
\text { Black/African } \\
\text { American, } \\
\text { Lower-income }\end{array}$ & - \\
\hline Daly et al. (13) & 1st March 2021 & General & - & 6,035 & - & - & $64.80 \%$ & $\begin{array}{l}\text { Younger age, } \\
\text { Females, } \\
\text { Black/African } \\
\text { American, } \\
\text { Lower-income }\end{array}$ & - \\
\hline $\begin{array}{l}\text { Mascarenhas et al. } \\
\text { (41) }\end{array}$ & 2020 & Dental students & $\begin{array}{l}\text { Michigan, } \\
\text { Florida, and Utah }\end{array}$ & 248 & $58 \%$ & $55.50 \%$ & $83.70 \%$ & - & - \\
\hline Marquez et al. (42) & $\begin{array}{l}\text { 28th } \\
\text { September-10th } \\
\text { November } 2020\end{array}$ & $\begin{array}{l}\text { Caregivers whose } \\
\text { children were being } \\
\text { treated in the dental } \\
\text { clinic at Cincinnati } \\
\text { Children's Hospital } \\
\text { Medical Center }\end{array}$ & Cincinnati, Ohio & 99 & $83.50 \%$ & $72.90 \%$ & $60.80 \%$ & - & - \\
\hline Sutton et al. (43) & $\begin{array}{l}\text { 7th-29th January } \\
2021\end{array}$ & $\begin{array}{l}\text { All women at the } \\
\text { Department of } \\
\text { Obstetrics and } \\
\text { Gynecology, Columbia } \\
\text { University Medical } \\
\text { Center }\end{array}$ & $\begin{array}{l}\text { New York City, } \\
\text { NY }\end{array}$ & 1,012 & $100 \%$ & $46.90 \%$ & $61 \%$ & Pregnant & Non-pregnant \\
\hline Kelekar et al. (44) & $\begin{array}{l}\text { September- } \\
\text { December } \\
2020\end{array}$ & $\begin{array}{l}3 \text { US dental schools } \\
\text { and } 1 \text { US medical } \\
\text { school }\end{array}$ & $\begin{array}{l}\text { Michigan, } \\
\text { Florida, and Utah }\end{array}$ & 415 & - & - & $63.85 \%$ & Dental students & $\begin{array}{l}\text { Medical } \\
\text { students }\end{array}$ \\
\hline Chin et al. (45) & $\begin{array}{l}\text { 22nd } \\
\text { December-4th } \\
\text { March } 2021\end{array}$ & State prison & California & 97,779 & - & - & $66.50 \%$ & $\begin{array}{l}\text { Black/African } \\
\text { American }\end{array}$ & - \\
\hline Levy et al. (46) & $\begin{array}{l}\text { 14th December } \\
\text { 2020-14th January } \\
2021\end{array}$ & Pregnant women & $\begin{array}{l}\text { New York City, } \\
\text { NY }\end{array}$ & 662 & $100 \%$ & - & $58.35 \%$ & $\begin{array}{l}\text { Risk to fetus, side } \\
\text { effects, } \\
\text { Black/African } \\
\text { American }\end{array}$ & $\begin{array}{l}\text { Non-Hispanic } \\
\text { white }\end{array}$ \\
\hline Ruggiero et al. (47) & - & General & - & 427 & - & - & $44.00 \%$ & $\begin{array}{l}\text { Fear of vaccines, } \\
\text { being against all } \\
\text { forms of } \\
\text { vaccines, } \\
\text { religious reasons }\end{array}$ & - \\
\hline $\begin{array}{l}\text { Doherty et al. } \\
\text { (preprint) (48) }\end{array}$ & $\begin{array}{l}\text { 27th August-15th } \\
\text { December } 2020\end{array}$ & General & North Carolina & 948 & 63 & $27.7 \%$ & $68.90 \%$ & $\begin{array}{l}\text { Black/African } \\
\text { American, safety } \\
\text { concerns }\end{array}$ & - \\
\hline
\end{tabular}


TABLE 1 | Continued

\begin{tabular}{|c|c|c|c|c|c|c|c|c|c|}
\hline Study & Study period & $\begin{array}{l}\text { Population } \\
\text { type/Settings }\end{array}$ & Region & $\begin{array}{l}\text { Sample size } \\
\text { (N) }\end{array}$ & Female sex $\%$ & $\begin{array}{l}\text { White } \\
\text { population \% }\end{array}$ & $\begin{array}{l}\text { Acceptance } \\
\text { rate } \%\end{array}$ & $\begin{array}{l}\text { Predictors of } \\
\text { hesitance }\end{array}$ & $\begin{array}{l}\text { Predictors of } \\
\text { acceptance }\end{array}$ \\
\hline Silva et al. (49) & November 2020 & $\begin{array}{l}\text { Student at Health } \\
\text { Services and College of } \\
\text { Pharmacy attending } \\
\text { influenza clinics }\end{array}$ & Rhode Island & 237 & $65 \%$ & $84 \%$ & $50 \%$ & $\begin{array}{l}\text { Safety, } \\
\text { effectiveness, and } \\
\text { limited } \\
\text { information }\end{array}$ & - \\
\hline Trent et al. (50) & July-August 2020 & General & $\begin{array}{l}\text { New York City, } \\
\text { NY, and } \\
\text { Phoenix, Arizona }\end{array}$ & 1,704 & $53 \%$ & - & $\begin{array}{l}71 \% \text { NY; } 76 \% \\
\text { Phoenix }\end{array}$ & $\begin{array}{l}\text { Younger age, } \\
\text { females }\end{array}$ & - \\
\hline Silva et al. (20) & $\begin{array}{l}\text { 19th October 16th } \\
\text { December } 2020\end{array}$ & $\begin{array}{l}\text { Sexual and Gender } \\
\text { Minority Men and } \\
\text { Transgender Women }\end{array}$ & - & 1,350 & - & $25.90 \%$ & $7(3.12)$ & - & - \\
\hline Hou et al. (51) & $\begin{array}{l}\text { 13th June-31st July } \\
2020\end{array}$ & $\begin{array}{l}\text { Twitter posts } \\
\text { mentioning COVID } \\
\text { vaccine }\end{array}$ & $\begin{array}{l}\text { New York City, } \\
\text { NY }\end{array}$ & 1,568 & - & - & $36.40 \%$ & - & - \\
\hline $\begin{array}{l}\text { Nguyen et al. } \\
\text { (preprint) (52) }\end{array}$ & $\begin{array}{l}\text { 24th March } \\
\text { 2020-16th February } \\
2021\end{array}$ & General & - & 73,650 & - & - & $91.40 \%$ & $\begin{array}{l}\text { Younger age, } \\
\text { Females, } \\
\text { Black/African } \\
\text { American }\end{array}$ & - \\
\hline Geana et al. (53) & $\begin{array}{l}\text { 5th-25th March } \\
2021\end{array}$ & $\begin{array}{l}\text { Women recently } \\
\text { released from jail }\end{array}$ & Midwest USA & 25 & $100 \%$ & $80.00 \%$ & $20 \%$ & $\begin{array}{l}\text { The pace of } \\
\text { development, } \\
\text { adverse effects }\end{array}$ & - \\
\hline $\begin{array}{l}\text { Piltch-Loeb et al. } \\
\text { (54) }\end{array}$ & $\begin{array}{l}\text { 13th-23rd } \\
\text { December } 2020\end{array}$ & General & - & 2,650 & $46.10 \%$ & $66 \%$ & $19.70 \%$ & $\begin{array}{l}\text { Black/African } \\
\text { American, } \\
\text { females }\end{array}$ & $\begin{array}{l}\text { Non-Hispanic } \\
\text { whites, Males }\end{array}$ \\
\hline Meier et al. (21) & $\begin{array}{l}\text { 28th-30th October } \\
2020\end{array}$ & General & - & 1,054 & $51.20 \%$ & $75.20 \%$ & $5.12(1.98)$ & - & - \\
\hline Latkin et al. (55) & $\begin{array}{l}\text { March, May, July } \\
2020\end{array}$ & General & - & 592 & $56 \%$ & $79.40 \%$ & $59.10 \%$ & - & Males \\
\hline Scott et al. (56) & April 2020 & Amish families & Ohio & 391 & - & - & $25 \%$ & - & - \\
\hline Szilagyi et al. (12) & $\begin{array}{l}\text { 25th November-8th } \\
\text { December } 2020\end{array}$ & $\begin{array}{l}\text { Uninstitutionalized US } \\
\text { residents }\end{array}$ & - & 5,660 & - & - & $56.2 \%$ & $\begin{array}{l}\text { Female, } \\
\text { Black/African } \\
\text { American }\end{array}$ & $\begin{array}{l}\text { Asian, Males, } \\
\text { Older age }\end{array}$ \\
\hline Szilagyi et al. (12) & 1st-14th April 2021 & $\begin{array}{l}\text { Uninstitutionalized US } \\
\text { residents }\end{array}$ & - & 5,660 & - & - & $74.1 \%$ & $\begin{array}{l}\text { Female, } \\
\text { Black/African } \\
\text { American }\end{array}$ & $\begin{array}{l}\text { Asian, Males, } \\
\text { Older age }\end{array}$ \\
\hline $\begin{array}{l}\text { Callaghan et al. } \\
\text { (16) }\end{array}$ & $\begin{array}{l}\text { 28th May-8th June } \\
2020\end{array}$ & General & - & 5,009 & 51.5 & $60 \%$ & $68.90 \%$ & - & - \\
\hline $\begin{array}{l}\text { Mercadante and } \\
\text { Law (57) }\end{array}$ & $\begin{array}{l}\text { 23rd-29th October } \\
2020 .\end{array}$ & General & - & 525 & $49 \%$ & $66.10 \%$ & $66.70 \%$ & - & - \\
\hline Kociolek et al. (58) & $\begin{array}{l}\text { 21st } \\
\text { December-13th } \\
\text { January } 2021\end{array}$ & Children's hospital staff & Chicago, Illinois & 4,448 & $81.60 \%$ & & $59.80 \%$ & $\begin{array}{l}\text { Black/African } \\
\text { American }\end{array}$ & - \\
\hline
\end{tabular}


TABLE 1 | Continued

\begin{tabular}{|c|c|c|c|c|c|c|c|c|c|}
\hline Study & Study period & $\begin{array}{l}\text { Population } \\
\text { type/Settings }\end{array}$ & Region & $\begin{array}{l}\text { Sample size } \\
\text { (N) }\end{array}$ & Female sex \% & $\begin{array}{l}\text { White } \\
\text { population \% }\end{array}$ & $\begin{array}{l}\text { Acceptance } \\
\text { rate } \%\end{array}$ & $\begin{array}{l}\text { Predictors of } \\
\text { hesitance }\end{array}$ & $\begin{array}{l}\text { Predictors of } \\
\text { acceptance }\end{array}$ \\
\hline Ruiz and Bell (59) & $\begin{array}{l}\text { 15th-16th June } \\
2020\end{array}$ & General & - & 804 & $53.60 \%$ & $65.3 \%$ & $14.80 \%$ & - & $\begin{array}{l}\text { Males, } \\
\text { non-Hispanic } \\
\text { white, } \\
\text { democrats, } \\
\text { college- } \\
\text { educated }\end{array}$ \\
\hline Latkin et al. (60) & $\begin{array}{l}\text { 14th-18th May } \\
2020\end{array}$ & General & - & 1,043 & $70.10 \%$ & $69.40 \%$ & $53.60 \%$ & $\begin{array}{l}\text { Black/African } \\
\text { American }\end{array}$ & - \\
\hline $\begin{array}{l}\text { Keene Woods } \\
\text { et al. (61) }\end{array}$ & April-May 2020 & General & Kansas & 53 & $96.15 \%$ & $50.94 \%$ & $59.60 \%$ & - & - \\
\hline $\begin{array}{l}\text { Greenhawt et al. } \\
\text { (62) }\end{array}$ & April 2020 & General & - & 1,262 & - & - & $65.70 \%$ & - & - \\
\hline Fisher et al. (63) & $\begin{array}{l}\text { 16th-20th April } \\
2020\end{array}$ & General & - & 991 & $51.50 \%$ & $63.3 \%$ & $57.60 \%$ & $\begin{array}{l}\text { Younger age, } \\
\text { Black/African } \\
\text { American, } \\
\text { Females, lower } \\
\text { educational } \\
\text { attainment, not } \\
\text { having received } \\
\text { the influenza } \\
\text { vaccine, need for } \\
\text { more information, } \\
\text { anti-vaccine } \\
\text { attitudes or } \\
\text { beliefs, lack of } \\
\text { trust }\end{array}$ & - \\
\hline Dorman et al. (7) & $\begin{array}{l}\text { October-November } \\
2020\end{array}$ & General & - & 26,324 & $72.80 \%$ & $51.6 \%$ & 4.6 & $\begin{array}{l}\text { Females or } \\
\text { Hispanic or } \\
\text { Black/African } \\
\text { American }\end{array}$ & $\begin{array}{l}\text { Males, older } \\
\text { age, } \\
\text { non-Hispanic } \\
\text { whites or Asian }\end{array}$ \\
\hline $\begin{array}{l}\text { Khubchandani } \\
\text { et al. (64) }\end{array}$ & June 2020 & General & - & 1,878 & $52 \%$ & $74 \%$ & $78 \%$ & $\begin{array}{l}\text { Black/African } \\
\text { American, } \\
\text { Hispanics, lower } \\
\text { education and } \\
\text { incomes, rural } \\
\text { dwellers, people } \\
\text { in the } \\
\text { northeastern US, } \\
\text { Republicans }\end{array}$ & Asian, others \\
\hline Kelly et al. (10) & April 2020 & General & - & 2,279 & $52 \%$ & $78 \%$ & $75 \%$ & $\begin{array}{l}\text { Black/African } \\
\text { American } \\
\text { Females }\end{array}$ & $\begin{array}{l}\text { Hispanics, } \\
\text { non-Hispanic } \\
\text { whites, males }\end{array}$ \\
\hline
\end{tabular}

(Continued) 
TABLE 1 | Continued

\begin{tabular}{|c|c|c|c|c|c|c|c|c|c|}
\hline Study & Study period & $\begin{array}{l}\text { Population } \\
\text { type/Settings }\end{array}$ & Region & $\begin{array}{l}\text { Sample size } \\
\text { (N) }\end{array}$ & Female sex $\%$ & $\begin{array}{l}\text { White } \\
\text { population \% }\end{array}$ & $\begin{array}{l}\text { Acceptance } \\
\text { rate } \%\end{array}$ & $\begin{array}{l}\text { Predictors of } \\
\text { hesitance }\end{array}$ & $\begin{array}{l}\text { Predictors of } \\
\text { acceptance }\end{array}$ \\
\hline $\begin{array}{l}\text { Rungkitwattanakul } \\
\text { et al. (65) }\end{array}$ & - & $\begin{array}{l}\text { Patients receiving } \\
\text { dialysis treatment }\end{array}$ & - & 90 & $40 \%$ & (Black 83\%) & $49 \%$ & $\begin{array}{l}\text { Lack of trust in } \\
\text { the federal } \\
\text { government Lack } \\
\text { of information, } \\
\text { safety do not trust } \\
\text { the manufacturer } \\
\text { of the vaccine }\end{array}$ & - \\
\hline Kelkar et al. (66) & $\begin{array}{l}\text { 31st December } \\
\text { 2020-8th January } \\
2021\end{array}$ & Cancer patients & Florida & 205 & $79 \%$ & $82 \%$ & $71 \%$ & - & - \\
\hline Malik et al. (67) & May 2020 & General & - & 672 & $57 \%$ & $73 \%$ & $67 \%$ & $\begin{array}{l}\text { Females, } \\
\text { Black/African } \\
\text { American }\end{array}$ & $\begin{array}{l}\text { Males, older } \\
\text { age, } \\
\text { non-Hispanic } \\
\text { whites or } \\
\text { Asian, college } \\
\text { degree }\end{array}$ \\
\hline Stern et al. (68) & $\begin{array}{l}\text { September- } \\
\text { December } \\
2020\end{array}$ & $\begin{array}{l}\text { Residents in three } \\
\text { prisons and } 13 \text { jails in } \\
\text { four states }\end{array}$ & $\begin{array}{l}\text { Washington, } \\
\text { Florida, } \\
\text { California, Texas }\end{array}$ & 5,110 & $17.60 \%$ & $41.90 \%$ & $44.90 \%$ & $\begin{array}{l}\text { Black/African } \\
\text { American, } \\
\text { Younger age, } \\
\text { lived in jails vs. } \\
\text { prisons waiting } \\
\text { for more } \\
\text { information, } \\
\text { efficacy, or safety } \\
\text { concerns }\end{array}$ & - \\
\hline Pogue et al. (69) & - & General & - & 316 & $49.38 \%$ & $63.27 \%$ & $68.54 \%$ & - & - \\
\hline Salmon et al. (70) & $\begin{array}{l}\text { 25th November-7th } \\
\text { December } 2020\end{array}$ & General & - & 2,525 & $51.80 \%$ & $39.70 \%$ & $50 \%$ & $\begin{array}{l}\text { Females, } \\
\text { black/African } \\
\text { American }\end{array}$ & $\begin{array}{l}\text { Males, } \\
\text { non-Hispanic } \\
\text { whites }\end{array}$ \\
\hline Unroe et al. (71) & $\begin{array}{l}\text { 14th-17th } \\
\text { November } 2020\end{array}$ & Nursing home staff & Indiana & 8,243 & $87 \%$ & $82.60 \%$ & $45 \%$ & $\begin{array}{l}\text { Side effects, } \\
\text { females, } \\
\text { Black/African } \\
\text { American }\end{array}$ & $\begin{array}{l}\text { Males, } \\
\text { non-Hispanic } \\
\text { whites }\end{array}$ \\
\hline Rhodes et al. (22) & 2020 & $\begin{array}{l}\text { Vaccine hesitant } \\
\text { parents }\end{array}$ & - & 1,381 & $61.80 \%$ & $68.80 \%$ & $3.58(2.16)$ & $\begin{array}{l}\text { General } \\
\text { unwillingness }\end{array}$ & $\begin{array}{l}\text { More } \\
\text { educated } \\
\text { respondents }\end{array}$ \\
\hline Lucia et al. (72) & - & Medical students & $\begin{array}{l}\text { Southeast } \\
\text { Michigan }\end{array}$ & 167 & $57 \%$ & - & $75.40 \%$ & - & - \\
\hline
\end{tabular}

(Continued) 


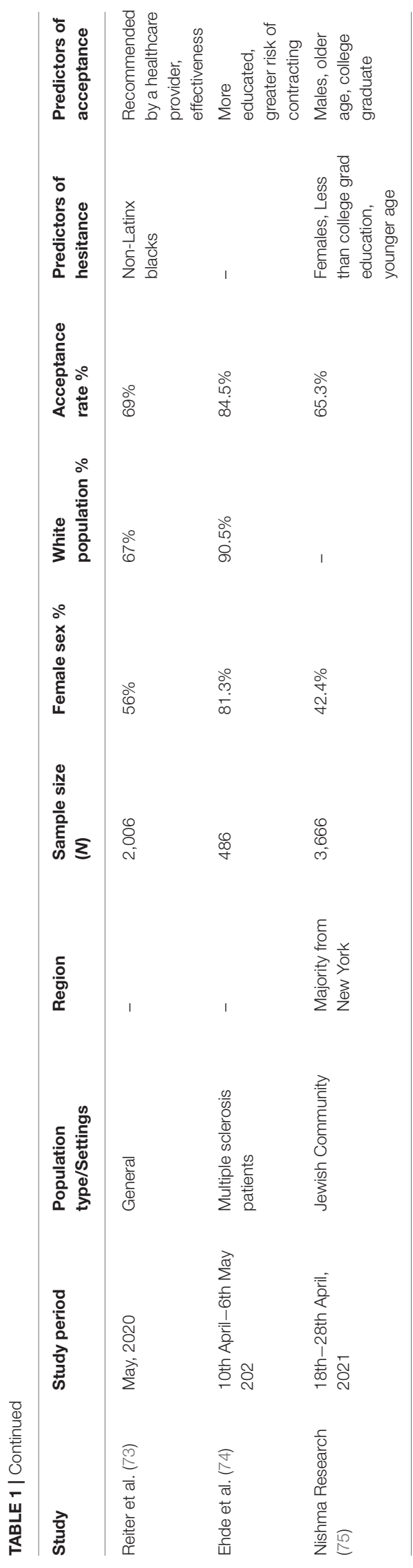

in the Mid Atlantic division of Northeast, all three states had surveys conducted, making it the only division with 100 percent data availability. Supplementary Table 1 states the percentage acceptance by each state, with Kansas reporting a high of $89.6 \%$.

\section{COVID-19 Vaccine Acceptance Stratified per Respondents' Population}

Apart from the studies with the general population, 18 surveys took place in a healthcare setting, including patients, workers and, students. From these 18, eight highlighted patients' attitudes toward the COVID-19 vaccine. Amongst patients, two surveys on solid organ transplant (SOT) recipients showed their hesitance toward the vaccine owing to lack of data and concerns of its safety in transplant recipients (56.10 and $31.63 \%$ acceptance). Other than SOT recipients, studies report willingness in patients with chronic disease, multiple sclerosis, dialysis, and cancer which showed a positive response toward the COVID-19 vaccine. Of these, patients suffering from chronic disease reported the highest acceptance rate of $85 \%$. Healthcare workers and students (dental and medical) showed a varying attitude toward the vaccine as acceptance ranged from a low of $45 \%$ in nursing home staff to a high of $90.10 \%$ in staff and students at the University of Kansas Medical Centre.

Three surveys were carried out in religious communities, two in Jewish and one in the Amish community. The Amish community had an alarmingly low acceptance rate of $25 \%$, whereas the Jewish people had 12 and $65.3 \%$, with the latter being of greater significance due to the large sample size. Two surveys carried out in New York highlighted the increased hesitance toward the vaccine by pregnant women. Amongst pregnant women, an acceptance rate of 44.3 and $58.35 \%$ was reported, whereas $76.2 \%$ of non-pregnant women were inclined to take up the COVID-19 vaccine. Studies on vaccine acceptance in inmates and those discharged from prison found a 20, 44.9, and, $66.5 \%$ acceptance rate in women recently released from prison, inmates from four states, and a California state prison, respectively, with the latter being significant with a sample size of 97,779. Furthermore, a survey on tobacco and marijuana users showed a low vaccine uptake rate of $49.1 \%$. In all four of these studies, Blacks/African Americans were correlated with increased unwillingness toward the COVID-19 vaccine. Figure 3 summarizes the percentage of vaccine acceptance in different population groups. Figure 4 demonstrates the pooled prevalence of COVID-19 vaccine acceptance in the United States with the pooled prevalence being $71.42 \%$.

\section{Factors Associated With Vaccine Acceptance and Hesitance}

Several factors were identified as leading to low or high vaccine acceptance. From the 65 studies, sex was mentioned in 22 surveys, race in 31 , age in 16, education level in 19, and income status in 4 . The male sex and individuals with a college degree or higher education were significantly associated with higher vaccine acceptance rates in all surveys reporting these variables. Furthermore, non-Hispanic Blacks were correlated with having the lowest vaccine acceptance in all 31 surveys whereas Whites 


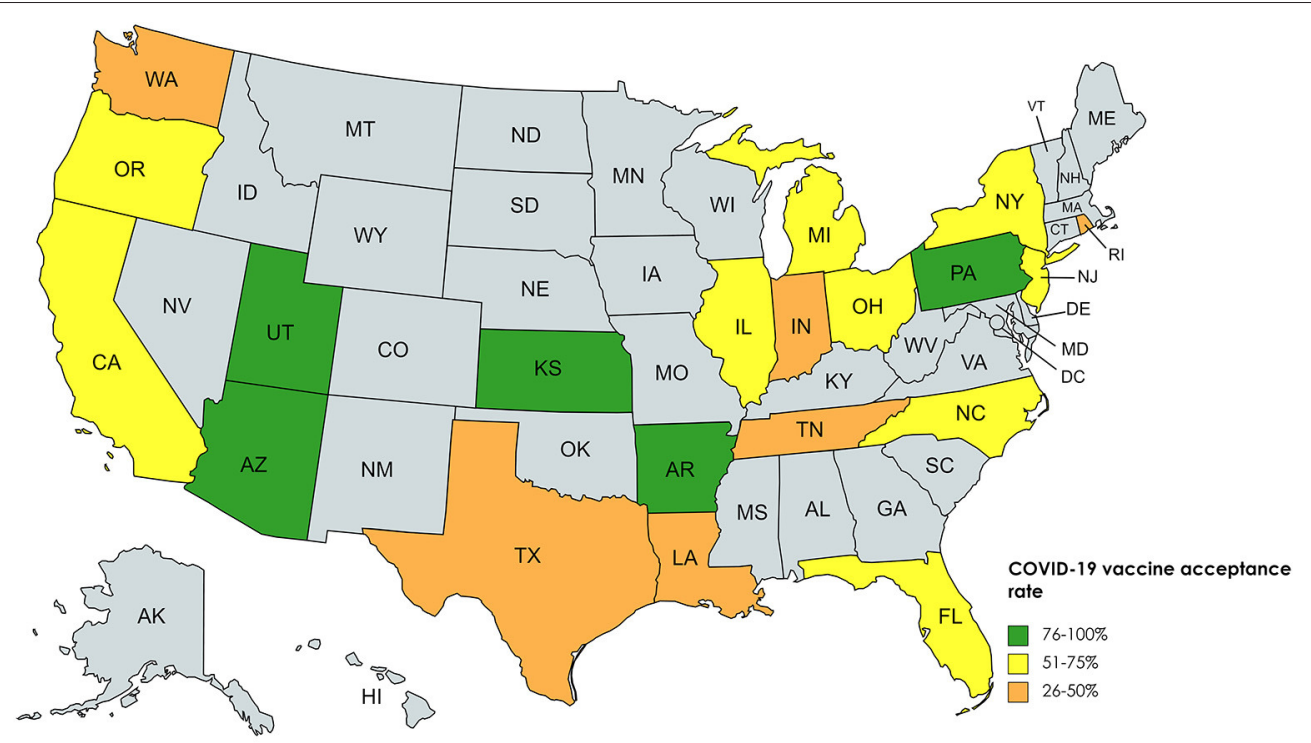

FIGURE 2 | COVID-19 vaccine acceptance rates of United States.

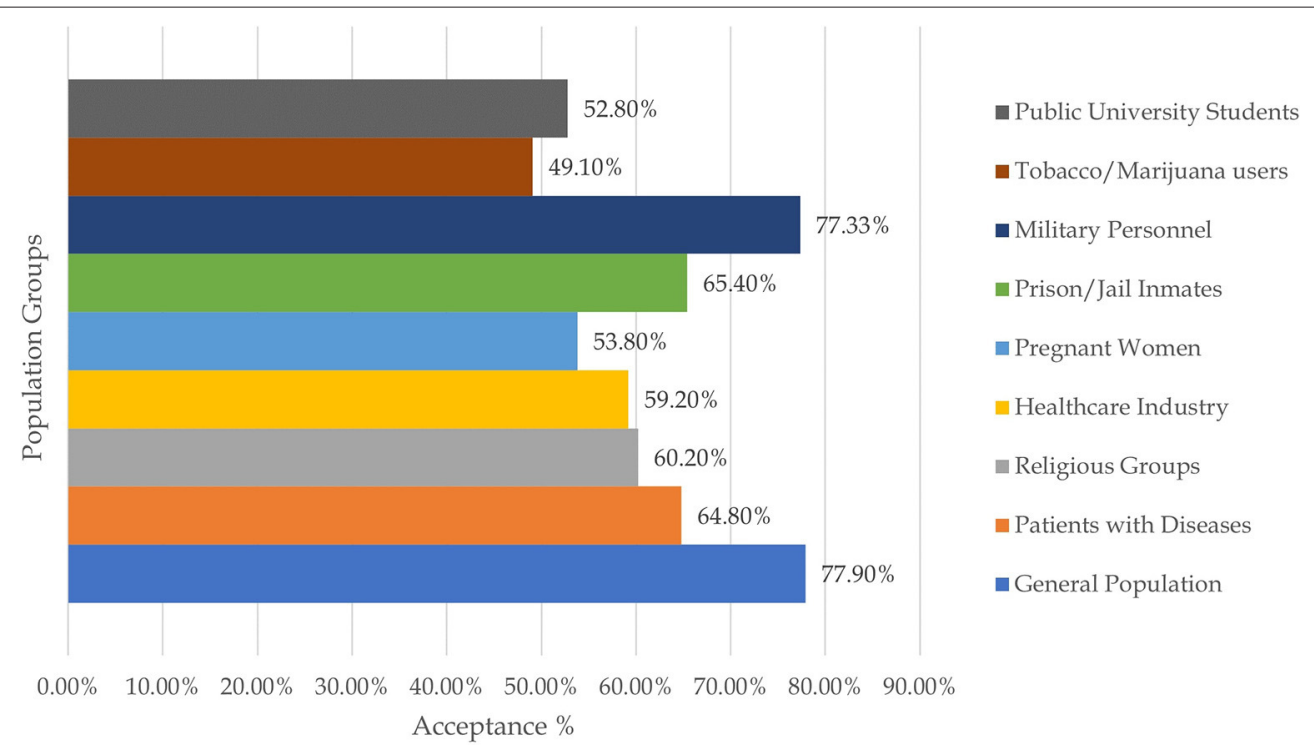

FIGURE 3 | COVID-19 vaccine acceptance stratified per-respondents' population.

and Asians showed a positive attitude toward uptake of the COVID-19 vaccination. People aged $>45$ years were linked with an increased approval of the vaccine compared to the younger population in 15 out of the 16 surveys. Respondents from lowerincome backgrounds were less inclined to get vaccinated in all four studies. Apart from these significant predictors of behavior toward a vaccine, other factors that contributed to vaccine hesitancy included uncertainty about the vaccine safety and side effects entailing after its administration, religious reasons, and a lack of trust in the healthcare system. A comprehensive data evaluation of the studies is included in Table 1.

\section{Changes in COVID-19 Vaccine Acceptance Over Time}

Two studies investigated the change in acceptance of the COVID19 vaccine by surveying the population twice. Daly et al. 


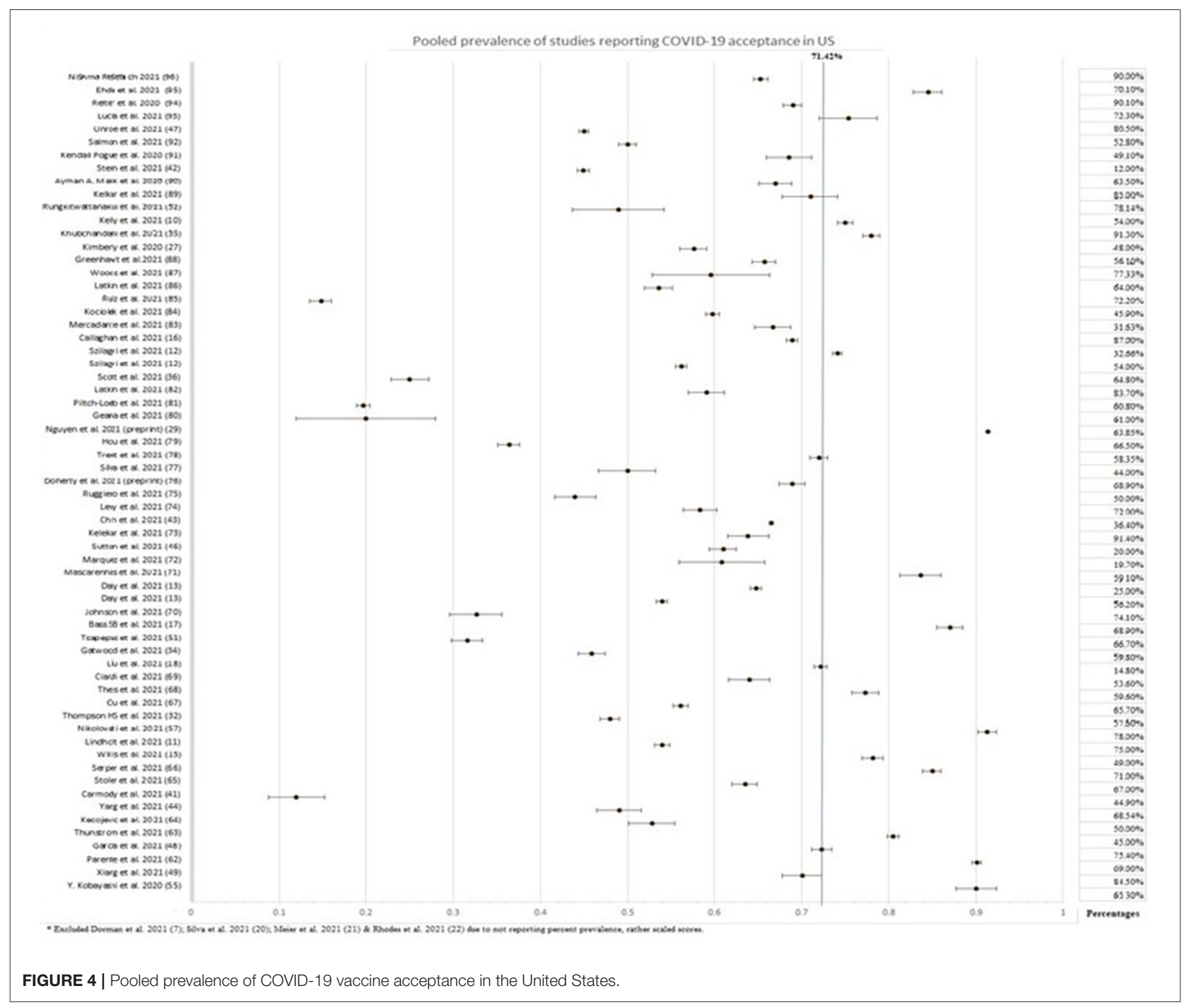

conducted the first round of the survey in October 2020, followed by the second one in March 2021. Percentage acceptance rose by $10.8 \%$ from 54 to $64.8 \%$. Furthermore, Szilagyi et al. carried out their surveys in November-December 2020 and April 2021 with 56.2 and $74.1 \%$ accepting the vaccine, respectively, marking a rise of $7.9 \%$. However, an overall change in readiness for uptake of the vaccine was notable; the predictors of hesitance remained the same throughout time. African/American Blacks and females were the leading predictors of low acceptance in both studies. Daly et al. also correlated younger age and lower-income with the increased unwillingness of the COVID-19 vaccine.

\section{DISCUSSION}

The emergence of the B.1.617.2 (Delta) variant has put the United States on the forefront in the total number of COVID19 cases countrywide (76). Studies have shown that a two-dose regime of any available vaccine significantly reduces a poor prognosis of COVID-19 (77). The rapid advent of efficacious vaccines for coronavirus is a projection of leaps in the field of medicine toward the goal of herd immunity. This, however, is threatened by the globally persistent but a re-emerging phenomenon of vaccine hesitancy and anti-vaccination, as experienced for vaccine-preventable diseases such as poliovirus and rubella. Despite a population of $59.9 \%$ receiving at least one dose of the COVID-19 vaccine (78), analyzing vaccine hesitancy is imperative to strategize vaccine delivery plans to procure maximum vaccine coverage and limit the spread of pathogens to the immunocompromised individuals and children who cannot be vaccinated.

In this systematic review, predictors of vaccine hesitancy and acceptance are studied across the U.S in the general population, pregnant women, immunocompromised individuals, students, healthcare workers, racial groups, and demographic 
characteristics. Factors associated with vaccine hesitancy are vastly different before and after the availability of vaccines. Before the roll-out of COVID-19 vaccines in mid-December 2020, major concerns across all the population groups were focused on the safety, effectiveness, and cost of the vaccine $(38,63)$. As the death toll peaked in the US, and with more publicly available data of vaccine trials, there was a considerable trend shift in the attitudes toward receiving a vaccination (33).

Thirty-three studies from a pool of 62 gathered vaccine acceptance rates from the general population, ranging from $91.4 \%$ (52) to a surprising low of $4.6 \%$ (7). Besides the difference in the timeline of each study, hesitancy was mainly driven by the lack of education and understanding of the process of vaccine development. As pharmaceutical companies develop multiple vaccines for the emerging strains, the expedited process of approval has raised concerns about its effectiveness (52), explaining their preferred choice of mRNA vaccines as opposed to any available vaccines (18).

Eleven studies report ethical and racial groups' unwillingness which stems from the deep-seated mistrust in the healthcare system. A study by Willis et al. conducted between July and August 2020 showed that one in every four Blacks/African Americans and Hispanics were hesitant to receive the COVID-19 vaccine, while COVID-19 vaccine hesitancy was highest among Black/African American respondents (50.00\%), followed by Hispanic/Latinx respondents (19.18\%), and White respondents (18.37\%) (15). An online survey from March 2021 revealed a significant decline in vaccine hesitancy amongst the Black race (14). Black/African Americans have been reported to consistently depict suspicion toward the vaccine, with similar patterns observed previously with the influenza vaccines. These can be traced back to the Tuskegee Syphilis study which mishandled hundreds of Black individuals and has institutionalized racial discrimination since then $(34,79)$. Consistent with the findings of observational studies, Blacks/African American race were more susceptible to SARS-CoV-2 than White Americans race; if contracted, the percentage of hospitalization and mortality was significantly higher (80). A survey that studied COVID19 vaccine acceptance in sexual and gender minority (SGM) group reported the least willingness in individuals who identified as Black; insensitivity from government representatives toward movements such as Black Lives Matter has deepened racial and economic disparity (20). Vaccine hesitancy in this minority group can be further explained by the pre-existing disparities with healthcare professionals, unavailability of healthcare services, and underrepresentation in clinical trials $(7,38,64)$. Maximum vaccine coverage can be achieved by building trust in the government and medical services; Black/African Americans and Hispanic healthcare workers can contribute to foster trust in the system. As many belong to the low-income strata, health insurance can help recover the losses. Providing maximum transparency in COVID-19 trials through informed consent, ensuring maximum representation, and consistent access to healthcare during and after the pandemic is likely to improve turnout at vaccination centers. Entrusting distribution and vaccine manufacturing to businesses owned by Blacks will be a step toward positive vaccine trends. However, Blacks and
Hispanics can only be freed from centuries of structural racism and classism in fields outside of medicine through consistent cultural policies across the US $(34,64,79)$.

Two studies report COVID-19 vaccine acceptance percentages in religious minorities in the US; an average willingness of $25 \%$ in Amish families of the Holmes County in Ohio (56) and $12 \%$ in Jewish Orthodox individuals in NY (30). Previous studies have shown that outbreaks of measles, rubella, and poliovirus have significantly affected the Amish community in Ohio, reflecting their lack of concern regarding the severity of the disease (81). Similar to findings of previous surveys conducted for Amish families, concerns about the adverse effects of COVID-19 vaccines were recognized as a major factor for vaccine hesitancy. Other predictors of unwillingness included avoiding dependence on the government, while some conservative families were not convinced as the bishop did not levy importance to it $(56,82)$. Local governments in Pennsylvania, Ohio, and Indiana have set up vaccination facilities at health departments to increase turnout (30). However, an effective approach to spreading COVID-19 vaccine awareness and destigmatizing it in the Amish community will have to be in conjunction with the church leadership, and the Old Order Amish families which were unaffected by the religious doctrine (82). On the contrary, shared accommodation amongst the Jewish community in NY has increased the incidence of COVID19, especially in the Chasidish sect. Regardless of the likelihood of contracting coronavirus, negative vaccine trends in Chasidish respondents in Brooklyn were observed (83). However, factors affecting their vaccination status; the belief that natural immunity was more beneficial, and the mistrust in physicians in the US, among other reasons put them on the priority list of strategizing distribution plans and approaches (30).

Individuals from ethnic minority groups or persons suffering from substance use disorders or mental illnesses are frequently incarcerated. The lack of stable housing, food supplies, and subpar treatment facilities delay diagnosis, which increases the risk of diseases like COVID-19 (68). Vaccine acceptance rates were relatively moderately high, ranging from 44 to $66 \%(45,68)$ in prisons while $49 \%$ in smokers (29). Consistent with previous surveys, hesitancy in prisoners originated from health illiteracy and the perception that a COVID-19 vaccine was unnecessary (68). In individuals who have been exposed to marijuana and/or tobacco, low acceptance was governed by demographic characteristics and if they lived alone or with a family of more than 5 people. This could be explained by the notion that living alone meant no exposure to SARS-CoV-2 while living with a larger family meant greater coronavirus exposure, and hence natural immunity (29). Although California, Washington, and Texas have recognized the importance of vaccinating detained residents and have put them on the priority list, distrust in government facilities revokes any efforts made.

As experienced in the $2009 \mathrm{H} 1 \mathrm{~N} 1$ influenza pandemic, a survey administered among reproductive-aged women from January 7, 2021, to January 29, 2021, established non-pregnant respondents to be most likely to accept vaccination, followed by breastfeeding responders with pregnant responders had the lowest vaccine acceptance (43). The lack of research of 
COVID-19 vaccines in pregnant women and females has raised major concerns related to its safety to the fetus, with many nonpregnant women considering disruption to the menstrual cycle and infertility a side effect (43). Future research should be geared toward pregnant and breastfeeding women who make up a large proportion of the population of the US.

Frontline healthcare workers and paramedics across the globe were the first to receive the COVID vaccine. Of the 9 studies reported in this systematic review, vaccine acceptance is one of the highest $(60-90 \%)$ in most studies. Nursing staff showed the least willingness to vaccination $(<50 \%)$ in multiple surveys which were conducted as vaccines were rolled out $(30,71)$. This results from the level of medical education in each group, and prospects about its long-term safety. The ethical duty of healthcare workers to not harm their patients makes relying on personal protective equipment insufficient and obligates them to urge staff and patients to receive the vaccine. COVID-19 poses a serious threat to immunocompromised patients, and upon hospitalization, such patients have an early mortality rate of $25 \%$ (26). In a population suffering from chronic illnesses, patients with multiple sclerosis have been included in trials, and thus show an acceptance rate between 60 and $70 \%(24,74)$. However, skepticism regarding vaccines was greatly observed in solid organ transplant (SOT) patients (31\% acceptance) (39) compared to hemodialysis patients (49\% acceptance) (65). SOT patients suffer from comorbidities such as chronic kidney disease and diabetes. Immunosuppressive drugs reduce hyper inflammation in SOT patients, but if infected with SARSCOV-2, risk bacterial and fungal co-infection as innate and adaptive immunity is compromised (84). Of the fundamental $5 \mathrm{Cs}$ that determine vaccine coverage, decreased confidence and medical mistrust correlated with the lack of research in SOT respondents (39). Suboptimal humoral responses were recorded with two-dose regimes of COVID-19 vaccines in organ transplant recipients. Therefore, three doses of COVID19 vaccines are being recommended to obtain recommended antibody titers (85).

The vaccine acceptance rates in the US vary by demographic characteristics, including age, geographical location, sex, and household income. While the country of origin of the vaccine has minimal contribution in encouraging or dissuading people from vaccination (23), statistics from March 2021 relay that vaccine hesitancy in the US was highest in low socioeconomic respondents belonging to households earning $\$ 50,000$ or less, among those without a degree adults, or those aged 18-39 years (13). Significant generational difference in vaccine hesitancy is shown as Baby Boomers and Gen X have lower odds of vaccine hesitancy compared to Millennials (86). In a study from 18 to 64-year-old Tennessee adults, a positive response was received regarding vaccine safety, efficacy, importance, and benefit (38). In a different clinical study comprising U.S. adults aged 65 and older, $63.6 \%$ of participants reported they were very willing to receive a COVID-19 vaccine (33). This is possibly governed by the social media exposure to each generation; Gen $\mathrm{Z}$ and Millennials are more likely to be technologically advanced as compared to Baby Boomer and Gen X. Therefore, health information could be easily obtained from social media platforms such as Facebook and
Twitter. A skewed display of factually incorrect information on social media and an individual's tendency to be vulnerable to social media's emotional appeal often becomes the gist of the decision made by a lot of individuals (87). Furthermore, a positive trend in vaccine acceptance among males vs. females (71), could be a result of a sampling bias thereof.

Medical mistrust is likely to improve if public health campaigns are run by health experts, CDC, or the WHO. Endorsements of COVID-19 vaccines by political figures is seen as politicizing a requirement of today, such as promotion of unapproved hydroxychloroquine by the US president wreaked havoc amongst the healthcare workers (88). Mandating vaccination in workplaces but also banning entry of unvaccinated individuals to public spaces may contribute as a factor of vaccine acceptance (89). Given the disproportionate willingness in the low-economic strata, efforts should be made to make the vaccine more accessible to them, by promoting health insurance. A study revealed only $35 \%$ of individuals were willing to pay $\$ 50$ or more for the coronavirus vaccine. Therefore, adopting a unified policy of a no-cost follow-up after vaccination as introduced by a few states can remove financial barriers (73). The expedited process of COVID-19 vaccine testing and the cases of adverse events of a vaccine contributed significantly to vaccine hesitance. This mandates the role of media and pharmaceutical companies to deliver information that can be easily interpreted by the general population (88).

This systematic review reports a multitude of populations, and analyses percentages by regions in the US. However, the pooled percentages are subjected to spectrum bias since percentages for general population are pooled with terminally ill or marginalized groups. Percentage of COVID-19 vaccine acceptance is liable to random error as published studies were carried out at different phases of the coronavirus peak. Another limitation worth noting is that it does not provide a timeline change in the vaccine hesitancy across all groups, and thus does not record the change in vaccine enthusiasm. Lastly, vaccine acceptance percentages by states should be interpreted logically as demographic characteristics differ across the region.

\section{CONCLUSION}

This study confirms that demographic characteristics played an important role in positive trends in vaccine acceptance, with the male sex showing higher acceptance of the COVID-19 vaccine. Least willingness was identified in the Black/African American population, and pregnant or breastfeeding women. Therefore, pharmaceutical companies and the US government must acknowledge the COVID-19 vaccine hesitancy that exists across the country. Increased hospitalization and adverse effects of COVID-19 in non-vaccinated individuals identify as potential threats to the economic and social stability of the country and aim to increase the divide. The willingness rates of vaccination are alarming in ethnic and sexual and gender minority groups, urging the need for outreach campaigns to prevent a COVID-19 outbreak in a susceptible population. Restoring trust in medical professionals and vaccines requires a strategical approach to 
overcome the racial injustice in the system, while timely implementation of vaccine delivery plans remains a challenge as new strains of coronavirus emerge as potential threats to the healthcare system.

\section{DATA AVAILABILITY STATEMENT}

The original contributions presented in the study are included in the article/Supplementary Material, further inquiries can be directed to the corresponding author/s.

\section{AUTHOR CONTRIBUTIONS}

FY: conception of the study, primary drafting of the work, final approval, and agreeing to the accuracy of the work. $\mathrm{HN}$ : conception of the study, major drafting of the work,

\section{REFERENCES}

1. Harrison EA, Wu JW. Vaccine confidence in the time of COVID-19. Eur J Epidemiol. (2020) 35:325-30. doi: 10.1007/s10654-020-00634-3

2. Chou WYS, Budenz A. Considering emotion in COVID19 vaccine communication: addressing vaccine hesitancy and fostering vaccine confidence. Health Commun. (2020) 35:1718-22. doi: $10.1080 / 10410236.2020 .1838096$

3. Different COVID-19 Vaccines. CDC. Available online at: https://www.cdc.gov/ coronavirus/2019-ncov/vaccines/different-vaccines.html (accessed August $12,2021)$.

4. Thunström L, Ashworth M, Finnoff D, Newbold SC. Hesitancy toward a COVID-19 vaccine. Ecohealth. (2021) 18:44-60. doi: 10.1007/s10393-021-01524-0

5. Coustasse A, Kimble C, Maxik K. COVID-19 and vaccine hesitancy: a challenge the united states must overcome. J Ambul Care Manage. (2021) 44:71-5. doi: 10.1097/JAC.0000000000000360

6. Painter EM. Demographic characteristics of persons vaccinated during the first month of the COVID-19 vaccination program - United States, December 14, 2020-January 14, 2021. MMWR Morb Mortal Wkly Rep. (2021) 70:174-7. doi: 10.15585/mmwr.mm7005e1

7. Dorman C, Perera A, Condon C, Chau C, Qian J, Kalk K, et al. Factors associated with willingness to be vaccinated against COVID-19 in a large convenience sample. J Commun Health. (2021) 46:1013-9. doi: 10.1007/s10900-021-00987-0

8. McAteer J, Yildirim I, Chahroudi A. The VACCINES act: deciphering vaccine hesitancy in the time of COVID-19. Clin Infect Dis. (2020) 71:703-5. doi: $10.1093 / \mathrm{cid} / \mathrm{ciaa} 433$

9. Stolle LB, Nalamasu R, Pergolizzi JV Jr., Magnusson P, LeQuang J, et al. Fact vs fallacy: the anti-vaccine discussion reloaded. $A d v$ Ther. (2020) 37:4481-90. doi: 10.1007/s12325-020-01502-y

10. Kelly BJ, Southwell BG, McCormack LA, Bann CM, MacDonald PDM, Frasier AM, et al. Predictors of willingness to get a COVID-19 vaccine in the U.S. BMC Infect Dis. (2021) 21:338. doi: 10.1186/s12879-021-06023-9

11. Lindholt MF, Jørgensen F, Bor A, Petersen MB. Public acceptance of COVID-19 vaccines: cross-national evidence on levels and individuallevel predictors using observational data. BMJ Open. (2021) 11:e048172. doi: 10.1136/bmjopen-2020-048172

12. Szilagyi PG, Thomas K, Shah MD, Vizueta N, Cui Y, Vangala S, et al. National trends in the US public's likelihood of getting a COVID-19 vaccine-April 1 to December 8, 2020. JAMA. (2021) 325:396-8. doi: 10.1001/jama.2020.26419

13. Daly $\mathrm{M}$, Jones $\mathrm{A}$, Robinson $\mathrm{E}$. Public trust and willingness to vaccinate against COVID-19 in the US from October 14, 2020, to March 29, 2021. JAMA. (2021) 325:2397-9. doi: 10.1001/jama.2021.8246 final approval, and agreeing to the accuracy of the work. AM: conception of the study, drafting of the work, final approval, and agreeing to the accuracy of the work. UN: drafting of the work, final approval, and agreeing to the accuracy of the work. MA: conception of the study, critical revision of the work, final approval, and agreeing to the accuracy of the work. NC, ZY, BS, IU, C-YL, and AP: critical revision of the work, final approval, and agreeing to the accuracy of the work. All authors have read and approved the final manuscript.

\section{SUPPLEMENTARY MATERIAL}

The Supplementary Material for this article can be found online at: https://www.frontiersin.org/articles/10.3389/fpubh. 2021.770985/full\#supplementary-material

14. Lin C, Tu P, Beitsch LM. Confidence and receptivity for COVID-19 vaccines: a rapid systematic review. Vaccines. (2020) 9:16. doi: 10.3390/vaccines 9010016

15. Willis DE, Andersen JA, Bryant-Moore K, et al. COVID-19 vaccine hesitancy: Race/ethnicity, trust, and fear. Clin Transl Sci. (2021) 1-8. doi: $10.1111 /$ cts. 13077

16. Callaghan T, Moghtaderi A, Lueck JA, Hotez P, Strych U, Dor A, et al. Correlates and disparities of intention to vaccinate against COVID-19. Soc Sci Med. (2021) 272:113638. doi: 10.1016/j.socscimed.2020.113638

17. Bass SB, Wilson-Genderson M, Garcia DT, Akinkugbe AA, Mosavel M. SARSCoV-2 vaccine hesitancy in a sample of us adults: role of perceived satisfaction with health, access to healthcare, and attention to COVID-19 news. Front Public Heal. (2021) 9:665724. doi: 10.3389/fpubh.2021.665724

18. Liu T, He Z, Huang J, Yan N, Chen Q, Huang F, et al. A comparison of vaccine hesitancy of COVID-19 vaccination in China and the United States. Vaccines. (2021) 9:649. doi: 10.3390/vaccines9060649

19. Page MJ, McKenzie JE, Bossuyt PM, Boutron I, Hoffmann TC, Mulrow CD, et al. The PRISMA 2020 statement: an updated guideline for reporting systematic reviews. BMJ. (2021) 372:n71. doi: 10.1136/bmj.n71

20. Silva DT da, Biello K, Lin WY, Valente PK, Mayer KH, Hightow-Weidman $\mathrm{L}$, et al. COVID-19 vaccine acceptance among an online sample of sexual and gender minority men and transgender women. Vaccines. (2021) 9:1-10. doi: 10.3390/vaccines 9030204

21. Meier BP, Dillard AJ, Lappas CM. Predictors of the intention to receive a SARS-CoV-2 vaccine. J Public Health. (2021) fdab013. doi: 10.1093/pubmed/fdab013

22. Rhodes ME, Sundstrom B, Ritter E, McKeever BW, McKeever R. Preparing for a COVID-19 vaccine: a mixed methods study of vaccine hesitant parents. J Health Commun. (2020) 25:831-7. doi: 10.1080/10810730.2021.1871986

23. Kobayashi Y, Howell C, Heinrich T. Vaccine hesitancy, state bias, and Covid-19: evidence from a survey experiment using Phase-3 results announcement by BioNTech and Pfizer. Soc Sci Med. (2021) 282:114115. doi: 10.1016/j.socscimed.2021.114115

24. Xiang XM, Hollen C, Yang Q, Brumbach BH, Spain RI, Wooliscroft L. COVID-19 vaccination willingness among people with multiple sclerosis. Mult Scler J Exp Transl Clin. (2021) 7. doi: 10.1177/20552173211017159

25. Parente DJ, Ojo A, Gurley T, Le Master JW, Meyer M, Wild DM, et al. Acceptance of COVID-19 vaccination among health system personnel. J Am Board Fam Med. (2021) 34:498-522. doi: 10.3122/jabfm.2021.03.200541

26. Garcia P, Montez-Rath ME, Moore H, Flotte J, Fults C, Block MS, et al. SARS$\mathrm{CoV}-2$ vaccine acceptability in patients on hemodialysis: a nationwide survey. J Am Soc Nephrol. (2021) 32:1575-81. doi: 10.1681/ASN.2021010104

27. Thunstrom L, Ashworth M, Finnoff D, Newbold S. Hesitancy towards a COVID-19 vaccine and prospects for herd immunity. SSRN Electron J. (2020) doi: 10.2139/ssrn.3593098 
28. Kecojevic A, Basch CH, Sullivan M, Chen YT, Davi NK. COVID19 vaccination and intention to vaccinate among a sample of college students in New Jersey. J Commun Health. (2021) 11:179-85. doi: 10.1007/s10900-021-00992-3

29. Yang Y, Dobalian A, Ward KD. COVID-19 vaccine hesitancy and its determinants among adults with a history of tobacco or marijuana use. $J$ Commun Health. (2021) 1-9. doi: 10.1007/s10900-021-00993-2

30. Carmody ER, Zander D, Klein EJ, Mulligan MJ, Caplan AL. Knowledge and attitudes toward covid-19 and vaccines among a New York Haredi-Orthodox Jewish Community. J Commun Health. (2021) 1-9. doi: 10.1007/s10900-021-00995-0

31. Stoler J, Enders AM, Klofstad CA, Uscinski JE. The limits of medical trust in mitigating COVID-19 vaccine hesitancy among black Americans. J Gen Intern Med. (2021) 1-3. doi: 10.1007/s11606-021-06743-3

32. Serper M, Reddy KR, Bewtra M, Ahmad N, Mehta SJ. COVID-19 vaccine perceptions among patients with chronic disease in a large gastroenterology and hepatology practice. Am J Gastroenterol. (2021) 116:1345-9. doi: 10.14309/ajg.0000000000001270

33. Nikolovski J, Koldijk M, Weverling GJ, Spertus J, Turakhia M, Saxon $\mathrm{L}$, et al. Factors indicating intention to vaccinate with a COVID19 vaccine among older U.S. adults. PLoS ONE. (2021) 16:e0251963. doi: 10.1371/journal.pone. 0251963

34. Thompson HS, Manning M, Mitchell J, Kim S, Harper FWK, Cresswell $\mathrm{S}$, et al. Factors associated with racial/ethnic group-based medical mistrust and perspectives on COVID-19 vaccine trial participation and vaccine uptake in the US. JAMA Netw Open. (2021) 4:e2111629. doi: 10.1001/jamanetworkopen.2021.11629

35. Ou MT, Boyarsky BJ, Zeiser LB, Chiang TP-Y, Ruddy J, Rasmussen SEVP, et al. Kidney transplant recipient attitudes toward a SARS-CoV-2 vaccine. Transplant Direct. (2021) 7:e713. doi: 10.1097/TXD.0000000000001171

36. Theis SR, Li PC, Kelly D, Ocampo T, Berglund A, Morgan D, et al. Perceptions and concerns regarding COVID-19 vaccination in a military base population. Mil Med. (2021) 2021:usab230. doi: 10.1093/milmed/usab230

37. Ciardi F, Menon V, Jensen JL, Shariff MA, Pillai A, Venugopal U, et al. Knowledge, attitudes and perceptions of COVID-19 vaccination among healthcare workers of an inner-city hospital in New York. Vaccines. (2021) 9:516. doi: 10.3390/vaccines 9050516

38. Gatwood J, McKnight M, Fiscus M, Hohmeier KC, Chisholm-Burns M. Factors influencing likelihood of COVID-19 vaccination: a survey of Tennessee adults. Am J Health Syst Pharm. (2021) 78:879-89. doi: 10.1093/ajhp/zxab099

39. Tsapepas D, Husain SA, King KL, Burgos Y, Cohen DJ, Mohan S. Perspectives on COVID-19 vaccination among kidney and pancreas transplant recipients living in New York City. Am J Heal Pharm. (2021) zxab272. doi: 10.1093/ajhp/zxab272

40. Johnson KD, Akingbola O, Anderson J, Hart J, Chapple A, Woods C, et al. Combatting a "Twin-demic": a quantitative assessment of COVID-19 and influenza vaccine hesitancy in primary care patients. Heal Promot Perspect. (2021) 11:179-85. doi: 10.34172/hpp.2021.22

41. Mascarenhas AK, Lucia VC, Kelekar A, Afonso NM. Dental students' attitudes and hesitancy toward COVID-19 vaccine. J Dent Educ. (2021) 85:1504-10. doi: 10.1002/jdd. 12632

42. Marquez RR, Gosnell ES, Thikkurissy S, Schwartz SB, Cully JL. Caregiver acceptance of an anticipated COVID-19 vaccination. J Am Dent Assoc. (2021) 152:730-9. doi: 10.1016/j.adaj.2021.03.004

43. Sutton D, D'Alton M, Zhang Y, Kahe K, Cepin A, Goffman D, et al. COVID19 vaccine acceptance among pregnant, breastfeeding, and nonpregnant reproductive-aged women. Am J Obstet Gynecol MFM. (2021) 3:100403. doi: 10.1016/j.ajogmf.2021.100403

44. Kelekar AK, Lucia VC, Afonso NM, Mascarenhas AK. COVID-19 vaccine acceptance and hesitancy among dental and medical students. J Am Dent Assoc. (2021) 152:596-603. doi: 10.1016/j.adaj.2021.03.006

45. Chin ET, Leidner D, Ryckman T, Liu YE, Prince L, Alarid-Escudero F, et al. Covid-19 vaccine acceptance in california state prisons. N Engl J Med. (2021) 385:374-6. doi: 10.1056/NEJMc2105282

46. Levy AT, Singh S, Riley LE, Prabhu M. Acceptance of COVID-19 vaccination in pregnancy: a survey study. Am J Obstet Gynecol MFM. (2021) 3:100399. doi: 10.1016/j.ajogmf.2021.100399
47. Ruggiero KM, Wong J, Sweeney CF, Avola A, Auger A, Macaluso M, et al. Parents' intentions to vaccinate their children against COVID-19. J Pediatr Heal Care. (2021) 35:509-17. doi: 10.1016/j.pedhc.2021.04.005

48. Doherty IA, Pilkington W, Brown L, Billings V, Hoffler U, Paulin L, et al. COVID-19 vaccine hesitancy in underserved communities of North Carolina. medRxiv. (2021). doi: 10.1101/2021.02.21.21252163

49. Silva J, Bratberg J, Lemay V. COVID-19 and influenza vaccine hesitancy among college students. J Am Pharm Assoc. (2021). doi: 10.1016/j.japh.2021.05.009

50. Trent M, Seale H, Ahmad Chughtai A, Salmon D, Raina MacIntyre C. Trust in government, intention to vaccinate and COVID-19 vaccine hesitancy: a comparative survey of five large cities in the United States, United Kingdom, and Australia. Vaccine. (2021). doi: 10.1016/j.vaccine.2021.06.048

51. Hou Z, Tong Y, Du F, Lu L, Zhao S, Yu K, et al. Assessing covid-19 vaccine hesitancy, confidence, and public engagement:a global social listening study. $J$ Med Internet Res. (2021) 23:e27632. doi: 10.2196/27632

52. Nguyen LH, Joshi AD, Drew DA, Merino J, Ma W, Lo C-H, et al. Racial and ethnic differences in COVID-19 vaccine hesitancy and uptake. medRxiv. (2021). doi: 10.1101/2021.02.25.21252402

53. Geana M V., Anderson S, Ramaswamy M. COVID-19 vaccine hesitancy among women leaving jails: a qualitative study. Public Health Nurs. (2021) 38:892-96. doi: 10.1111/phn.12922

54. Piltch-Loeb R, Savoia E, Goldberg B, Hughes B, Verhey T, Kayyem J, et al. Examining the effect of information channel on COVID-19 vaccine acceptance. PLoS ONE. (2021) 16:e0251095. doi: 10.1371/journal.pone. 0251095

55. Latkin C, Dayton LA, Yi G, Konstantopoulos A, Park J, Maulsby C, et al. COVID-19 vaccine intentions in the United States, a social-ecological framework. Vaccine. (2021) 39:2288. doi: 10.1016/j.vaccine.2021.02.058

56. Scott EM, Stein R, Brown MF, Hershberger J, Scott EM, Wenger OK. Vaccination patterns of the northeast Ohio Amish revisited. Vaccine. (2021) 39:1058-63. doi: 10.1016/j.vaccine.2021.01.022

57. Mercadante AR, Law A V. Will they, or Won't they? Examining patients' vaccine intention for flu and COVID-19 using the Health Belief Model. Res Soc Adm Pharm. (2021) 17:1596. doi: 10.1016/j.sapharm.2020.12.012

58. Kociolek LK, Elhadary J, Jhaveri R, Patel AB, Stahulak B, Cartland J. Coronavirus disease 2019 vaccine hesitancy among children's hospital staff: a single-center survey. Infect Control Hosp Epidemiol. (2021) 42:775-7. doi: $10.1017 /$ ice. 2021.58

59. Ruiz JB, Bell RA. Predictors of intention to vaccinate against COVID19: results of a nationwide survey. Vaccine. (2021) 39:1080-6. doi: 10.1016/j.vaccine.2021.01.010

60. Latkin CA, Dayton L, Yi G, Colon B, Kong X. Mask usage, social distancing, racial, and gender correlates of COVID-19 vaccine intentions among adults in the US. PLoS ONE. (2021) 16:e0246970. doi: 10.1371/journal.pone.02 46970

61. Keene Woods N, Vargas I, McCray-Miller M, Drassen Ham A, Chesser AK. SARS-CoV2, the COVID-19 pandemic and community perceptions. J Prim Care Community Heal. (2021) 12. doi: 10.1177/21501327219 95451

62. Greenhawt M, Kimball S, DunnGalvin A, Abrams EM, Shaker MS, Mosnaim G, et al. Media influence on anxiety, health utility, and health beliefs early in the SARS-CoV-2 pandemic-a survey study. J Gen Intern Med. (2021) 36:1327. doi: 10.1007/s11606-020-06554-y

63. Fisher KA, Bloomstone SJ, Walder J, Crawford S, Fouayzi H, Mazor KM. Attitudes toward a potential SARS-CoV-2 vaccine : a survey of U.S. adults. Ann Intern Med. (2020) 173:964-73. doi: 10.7326/M20-3569

64. Khubchandani J, Sharma S, Price JH, Wiblishauser MJ, Sharma M, Webb FJ. COVID-19 vaccination hesitancy in the united states: a rapid national assessment. J Community Health. (2021) 46:270-7. doi: 10.1007/s10900-020-00958-x

65. Rungkitwattanakul D, Yabusaki A, Singh D, Lawson P, Nwaogwugwu U, Iheagwara OS, et al. COVID-19 vaccine hesitancy among African American hemodialysis patients: a single-center experience. Hemodial Int. (2021) 25:410-2. doi: 10.1111/hdi.12922

66. Kelkar AH, Blake JA, Cherabuddi K, Cornett H, McKee BL, Cogle CR. Vaccine enthusiasm and hesitancy in cancer patients and the impact of a webinar. Healthc. (2021) 9:351. doi: 10.3390/healthcare9030351 
67. Malik AA, McFadden SAM, Elharake J, Omer SB. Determinants of COVID19 vaccine acceptance in the US. EClinicalMedicine. (2020) 26:100495. doi: 10.1016/j.eclinm.2020.100495

68. Stern MF, Piasecki AM, Strick LB, Rajeshwar P, Tyagi E, Dolovich S, et al. Willingness to receive a COVID-19 vaccination among incarcerated or detained persons in correctional and detention facilities - four states, September-December (2020). MMWR Morb Mortal Wkly Rep. (2021) 70:473-7. doi: 10.15585/mmwr.mm7013a3

69. Pogue K, Jensen JL, Stancil CK, Ferguson DG, Hughes SJ, Mello EJ, et al. Influences on attitudes regarding potential covid-19 vaccination in the united states. Vaccines. (2020) 8:1-14. doi: 10.3390/vaccines80 40582

70. Salmon DA, Dudley MZ, Brewer J, Kan L, Gerber JE, Budigan H, et al. COVID-19 vaccination attitudes, values and intentions among United States adults prior to emergency use authorization. Vaccine. (2021) 39:2698-711. doi: $10.1016 /$ j.vaccine.2021.03.034

71. Unroe KT, Evans R, Weaver L, Rusyniak D, Blackburn J. Willingness of longterm care staff to receive a COVID-19 vaccine: a single state survey. J Am Geriatr Soc. (2021) 69:593-9. doi: 10.1111/jgs.17022

72. Lucia VC, Kelekar A, Afonso NM. COVID-19 vaccine hesitancy among medical students. J Public Health. (2020) 43:445-9. doi: 10.1093/pubmed/fdaa230

73. Reiter PL, Pennell ML, Katz ML. Acceptability of a COVID-19 vaccine among adults in the United States: how many people would get vaccinated? Vaccine. (2020) 38:6500-7. doi: 10.1016/j.vaccine.2020. 08.043

74. Ehde DM, Roberts MK, Herring TE, Alschuler KN. Willingness to obtain COVID-19 vaccination in adults with multiple sclerosis in the United States. Mult Scler Relat Disord. (2021) 49:102788. doi: 10.1016/j.msard.2021.1 02788

75. Nishma Research. COVID-19 Attitudes Vaccine Sentiment in the U.S. Orthodox Jewish Community: Views Among the Chasidish, Yeshivish, and Modern Orthodox Segments. (2021). Available online at: http:// nishmaresearch.com (accessed August 23, 2021).

76. Coronavirus Cases Worldwide by Country. Statista. Available online at: https://www.statista.com/statistics/1043366/novel-coronavirus-2019ncovcases-worldwide-by-country/ (accessed August 16, 2021).

77. Bernal JL, Andrews N, Gower C, Gallagher E, Simmons R, Thelwall S, et al. Effectiveness of Covid-19 vaccines against the B.1.617.2 (Delta) variant. N Engl J Med. (2021) 385:585-94. doi: 10.1056/NEJMoa2108891

78. U.S. COVID-19 Vaccine Tracker: See Your State's Progress. Mayo Clinic. Available online at: https://www.mayoclinic.org/coronavirus-covid-19/ vaccine-tracker (accessed August 17, 2021).

79. Warren RC, Forrow L, Hodge DA Sr., Truog RD. Trustworthiness before trust - covid-19 vaccine trials and the black community. N Engl J Med. (2020) 383:e121. doi: 10.1056/NEJMp2030033

80. Vahidy FS, Mph M, Carlos J, Bs N, Meeks JR, Khan Bs O, et al. Racial and ethnic disparities in SARS-CoV-2 pandemic: analysis of a COVID-19 observational registry for a diverse U.S. metropolitan population. BMJ Open. (2020) 10:e039849. doi: 10.1136/bmjopen-2020-039849
81. Yoder JS, Dworkin MS. Vaccination usage among an old-order Amish community in Illinois. Pediatr Infect Dis J. (2006) 25:1182-3. doi: 10.1097/01.inf.0000246851.19000.3e

82. Kettunen C, Nemecek J, Wenger O. Evaluation of low immunization coverage among the Amish population in rural Ohio. AJIC Am J Infect Control. (2017) 45:630-4. doi: 10.1016/j.ajic.2017.01.032

83. Nishma Research. COVID-19 Attitudes Vaccine Sentiment in the U.S Orthodox Jewish Community: Views Among the Chasidish, Yeshivish, and Modern Orthodox Segment. (2021). Available online at: http://nishmaresearch. com (accessed August 18, 2021).

84. Ao G, Wang Y, Qi X, Nasr B, Bao M, Gao M, et al. The association between severe or death COVID-19 and solid organ transplantation: a systematic review and meta-analysis. Transplant Rev. (2021) 35:100628. doi: $10.1016 /$ j.trre.2021.100628

85. Werbel WA, Boyarsky BJ, Ou MT, Massie AB, Tobian AAR, Garonzik-Wang $\mathrm{JM}$, et al. Safety and immunogenicity of a third dose of SARS-CoV-2 vaccine in solid organ transplant recipients: a case series. Ann Intern Med. (2021) 174:1330-2. doi: 10.7326/L21-0282

86. Shih S-F, Wagner AL, Masters NB, Prosser LA, Lu Y, ZikmundFisher BJ. Vaccine hesitancy and rejection of a vaccine for the novel coronavirus in the United States. Front Immunol. (2021). 12:558270. doi: 10.3389/fimmu.2021.558270

87. N P, EA C, H H, K G. Social media and vaccine hesitancy: new updates for the era of COVID-19 and globalized infectious diseases. Hum Vaccin Immunother. (2020) 16:2586-93. doi: 10.1080/21645515.2020.1780846

88. Bunch L. A tale of two crises: addressing covid-19 vaccine hesitancy as promoting racial justice. Hec Forum. (2021) 33:143-54. doi: 10.1007/s10730-021-09440-0

89. Vergara RJD, Sarmiento PJD, Lagman JDN. Building public trust: a response to COVID-19 vaccine hesitancy predicament. J Public Health. (2021) 43:e2912. doi: 10.1093/pubmed/fdaa282

Conflict of Interest: The authors declare that the research was conducted in the absence of any commercial or financial relationships that could be construed as a potential conflict of interest.

Publisher's Note: All claims expressed in this article are solely those of the authors and do not necessarily represent those of their affiliated organizations, or those of the publisher, the editors and the reviewers. Any product that may be evaluated in this article, or claim that may be made by its manufacturer, is not guaranteed or endorsed by the publisher.

Copyright () 2021 Yasmin, Najeeb, Moeed, Naeem, Asghar, Chughtai, Yousaf, Seboka, Ullah, Lin and Pakpour. This is an open-access article distributed under the terms of the Creative Commons Attribution License (CC BY). The use, distribution or reproduction in other forums is permitted, provided the original author $(s)$ and the copyright owner(s) are credited and that the original publication in this journal is cited, in accordance with accepted academic practice. No use, distribution or reproduction is permitted which does not comply with these terms. 